\title{
Interleukin (IL)-1 family cytokines could differentiate primary immune thrombocytopenia from systemic lupus erythematosus-associated thrombocytopenia
}

\author{
Yanxia Zhan ${ }^{1 \#}$, Luya Cheng ${ }^{1 \#}$, Boting $\mathrm{Wu}^{2}$, Lili Ji ${ }^{1}$, Pu Chen ${ }^{3}$, Feng $\mathrm{Li}^{1,4}$, Jingjing Cao ${ }^{1}$, Yang $\mathrm{Ke}^{1}$, \\ Ling Yuan', Zhihui Min ${ }^{5}$, Lihua Sun ${ }^{3}$, Hao Chen ${ }^{6}$, Fanli Hua ${ }^{4}$, Yunfeng Cheng ${ }^{1,3,5,7}$ \\ ${ }^{1}$ Department of Hematology, Zhongshan Hospital Fudan University, Shanghai, China; ${ }^{2}$ Department of Transfusion Medicine, Zhongshan Hospital \\ Fudan University, Shanghai, China; ${ }^{3}$ Department of Clinical Laboratory, Zhongshan Hospital Fudan University, Shanghai, China; ${ }^{4}$ Department of \\ Hematology, Zhongshan Hospital Qingpu Branch, Fudan University, Shanghai, China; ${ }^{5}$ Institute of Clinical Science, Zhongshan Hospital Fudan \\ University, Shanghai, China; ${ }^{6}$ Department of Thoracic Surgery, Zhongshan Hospital Xuhui Branch, Fudan University, Shanghai, China; ${ }^{7}$ Center for \\ Tumor Diagnosis \& Therapy, Jinshan Hospital, Fudan University, Shanghai, China \\ Contributions: (I) Conception and design: Y Zhan, B Wu, L Cheng, Y Cheng; (II) Administrative support: B Wu, F Li, Y Cheng; (III) Provision of \\ study materials or patients: L Cheng, L Ji, Y Ke, L Yuan, Y Cheng; (IV) Collection and assembly of data: Y Zhan, J Cao, P Chen, F Hua, Z Min, \\ L Ji, L Sun; (V) Data analysis and interpretation: Y Zhan, L Cheng, B Wu, H Chen, F Hua; (VI) Manuscript writing: All authors; (VII) Final \\ approval of manuscript: All authors. \\ "These authors contributed equally to this work. \\ Correspondence to: Yunfeng Cheng, MD, PhD; Fanli Hua, MD, PhD. Institute of Clinical Science, Department of Hematology, Zhongshan Hospital, \\ Fudan University, 180 Fenglin Rd., Shanghai 200032, China. Email: yfcheng@fudan.edu.cn; hua_fanli@fudan.edu.cn.
}

Background: Primary immune thrombocytopenia (ITP) is an autoimmune-mediated disorder characterized by a decreased platelet count. Systemic lupus erythematosus (SLE) is also an autoimmune disease in which thrombocytopenia is a common hematologic manifestation. Interleukin (IL)-1 family cytokines are major proinflammatory and immunoregulatory mediators. This study aimed to investigate the role of IL-1 cytokines in patients with ITP and SLE and the potential pathophysiologic mechanism to differentiate SLE-associated thrombocytopenia (SLE-TP) from ITP.

Methods: Multiplex cytokine assay and real-time polymerase chain reaction (RT-PCR) were used to measure IL-1 cytokines in 17 newly diagnosed ITP patients, 17 SLE-TP patients, 19 SLE patients without thrombocytopenia (SLE-NTP), and 10 healthy controls.

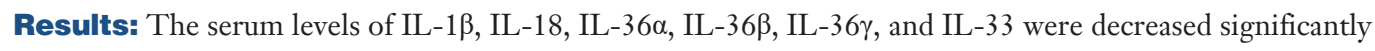
in ITP patients compared with SLE-TP patients, SLE-NTP patients, and healthy controls $(\mathrm{P}<0.05)$. While there was no significant difference in the serum level of IL-37 between ITP and SLE-TP patients, there was a positive correlation between the platelet count and IL-37 level in ITP patients. Our data suggested

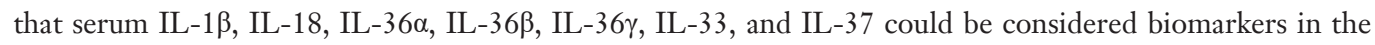
diagnosis of ITP.

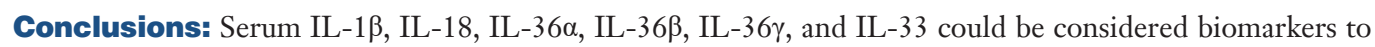
differentiate SLE-TP from ITP patients.

Keywords: Primary immune thrombocytopenia (primary ITP); systemic lupus erythematosus (SLE); interleukin-1 cytokine family; biomarker (IL-1 cytokine family; biomarker)

Submitted Jun 16, 2020. Accepted for publication Nov 20, 2020.

doi: $10.21037 / \mathrm{atm}-20-4729$

View this article at: http://dx.doi.org/10.21037/atm-20-4729 


\section{Introduction}

Primary immune thrombocytopenia (ITP) is an autoimmunemediated acquired bleeding disorder, defined as a platelet count less than $100 \times 10^{9} / \mathrm{L}$ without other causes of isolated thrombocytopenia $(1,2)$. The etiology of ITP is complex and heterogeneous, and as no specific biomarkers are indicating its presence, ITP remains a diagnosis of exclusion. The heterogeneous nature of ITP is evident in the differences in clinical presentation and response to regular treatment among patients and the multiple mechanisms that have been forwarded to account for it, such as autoantibodies, $\mathrm{T}$ cell dysregulation, and impaired megakaryocytes $(1,2)$. Except primary ITP, all forms of immune-mediated thrombocytopenias is defined as secondary ITP. Secondary ITP has several causes, including autoimmune diseases such as systemic lupus erythematosus (SLE).

SLE is a complex autoimmune disease and is usually associated with hematological abnormality (3), including thrombocytopenia, the prevalence of which in SLE patients has been reported to be 7-30\% (4). Conversely, the prevalence of SLE in all ITP cases in adults is approximately $5 \%$, making SLE the most common cause of secondary ITP (5). In the early stages, when there are only thrombocytopenia symptoms, it is sometimes difficult to determine what form of ITP is present in patients with SLE. SLE-associated thrombocytopenia (SLE$\mathrm{TP}$ ) is defined as a platelet count less than $100 \times 10^{9} / \mathrm{L}$ in the absence of any other identifiable cause (6). The pathogenesis of thrombocytopenia in SLE is heterogeneous and multifactorial. However, it is widely accepted that an increased platelet clearance mediated by autoantibodies against platelets contributes to the pathogenesis, which is analogous to the mechanism of ITP. Differing from primary ITP, the clinical treatment for thrombocytopenia secondary to an identifiable cause is often targeted to the ongoing disorder. However, there are no specific biomarkers to differentiate SLE-TP from ITP (7-9).

The family of interleukin (IL)-1 cytokines is a family of protein molecules consisting of 11 members, including IL-1 $\alpha$ (IL-1F1), IL-1 $\beta$ (IL-1F2), IL-1 receptor antagonist (IL-1Ra, IL-1F3), IL-18 (IL-1F4), IL-36Ra (IL-1F5), IL$36 \alpha$ (IL-1F6), IL-37 (IL-1F7), IL-36 $\beta$ (IL-1F8), IL-36 $\gamma$ (IL-1F9), IL-38 (IL-1F10), and IL-33 (IL-1F11) (10). This cytokione family plays a crucial role as major proinflammatory and immunoregulatory mediators in a wide range of autoinflammatory, infectious, tumor, and autoimmune diseases that act through the receptors of the Toll-like/IL-1 receptor superfamily (11-15). The production of inflammatory cytokines such as IL-1, IL18 , and IL-36 acts by activating target cells through the receptor superfamily then amplifying the immune response. However, antagonists such as IL-1Ra, the receptor antagonist of IL- $1 \alpha$ and IL- $1 \beta$, act as inhibitors of IL- 1 dependent inflammation.

The blocking of IL-1, particularly of IL- $1 \beta$, has recently become the standard therapy for autoinflammatory diseases $(16,17)$. Moreover, IL-1 $\beta$, a driver of tumor-promoting inflammation in cancer, can be targeted in patients using an IL-1 receptor antagonist acting as a checkpoint inhibitor $(18,19)$. Several studies have suggested abnormal changes in IL-18, and IL-18-binding protein (IL-18BP) were involved in the pathogenesis of SLE and ITP (20-23). Furthermore, recent studies demonstrate that IL-1 may also take part in inflammatory pathologies and auto-immune diseases by participating in the development of T-helper 17 (Th17) cells $(24,25)$, and increased numbers of Th17 cells have been reported in patients with SLE and ITP. We hypothesize that IL-1 family cytokines could be involved in the pathogenesis of ITP and/or SLE because of their role in inflammation and autoimmune diseases. In the present study, we aimed to evaluate IL-1 family cytokines' expression in patients with either ITP, SLE-TP, or those with SLE without thrombocytopenia (SLE-NTP), which might help to differentiate patients with SLE-TP from those with ITP. We present the following article following the MDAR reporting checklist (available at http://dx.doi. org/10.21037/atm-20-4729).

\section{Methods}

\section{Patients and controls}

We enrolled seventeen patients with newly diagnosed primary ITP (10 females and 7 males, age range 23-71 years, median age 52 years), seventeen patients with SLE-TP (15 females and 2 males, age range 21-61 years, median age 36 years), and nineteen patients with SLE-NTP (15 females and 4 males, age range $17-66$ years, median age 36 years). These patients were recruited from the Zhongshan Hospital of Fudan University (Shanghai, China) between July 2014 and May 2018, fulfilling the diagnosis criteria of ITP updated by an international working group (1) and the diagnosis of SLE according to the European League Against Rheumatism Executive Committee and the American College of Rheumatology criteria (6), respectively. A healthy 
control group composed of 10 age- and sex-matched healthy controls (6 females and 4 males, age range 24-68 years, median age 40.5 years) were also enrolled. The study was approved by the local Medical Ethics Committees of the Zhongshan Hospital of Fudan University (approval No. B2013-003R), and written informed consent to participate was obtained from each patient. The study was conducted following the Declaration of Helsinki (as revised in 2013).

\section{Multiplex cytokine assay}

Blood samples were obtained from ITP patients, SLE patients, and healthy controls. The samples were left to clot at room temperature for $1 \mathrm{~h}$, and serum was removed by centrifugation $(1,500 \mathrm{~g}, 10 \mathrm{~min})$ then stored at $-80^{\circ} \mathrm{C}$ until used. Serum levels of IL-1 family cytokines (IL-1 $\alpha$, IL-

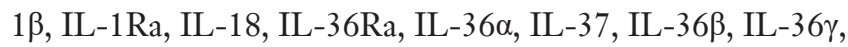
IL-38, and IL-33) were measured by Quantibody Human IL-1 Family Cytokine Array 1 (RayBiotech, USA), according to the manufacturer's protocol and the signals (green fluorescence, $\mathrm{Cy} 3$ channel, 555-nm excitation, and 565-nm emission) were captured using a LuxScan-10K/A (Capitalbio, China). Quantitative data analysis was performed using RayBio Q Analyzer Software (RayBiotech, USA).

\section{Isolation of peripheral blood mononuclear cells (PBMCs)}

Ethylenediaminetetraacetic acid (EDTA) anticoagulated venous blood samples were collected and diluted 1:2 with Hanks balanced salt solution (HBSS) before Ficoll-Hypaque gradient centrifugation $(2,500 \mathrm{rpm}$ at room temperature for $20 \mathrm{~min}$ ). Samples were then washed and resuspended in fetal bovine serum containing $10 \%$ dimethyl sulfoxide (DMSO) and stored at $-80^{\circ} \mathrm{C}$ until RNA extraction.

\section{Real-time polymerase chain reaction (RT-PCR)}

Total RNA was isolated from the PBMCs of each sample using Trizol reagent (Invitrogen, USA) and converted into cDNA using a PrimeScript RT Reagent Kit (Takara, Japan) following the manufacturer's instructions. The mRNA expressions of IL-1 cytokines were quantified using TB Green Premix Ex Taq (Takara, Japan) on a 7500 Real Time PCR System (Applied Biosystems, USA). Amplification was performed for 40 cycles of $5 \mathrm{~s}$ at $95^{\circ} \mathrm{C}$ and $34 \mathrm{~s}$ at $60^{\circ} \mathrm{C}$ after initial denaturation $\left(95^{\circ} \mathrm{C}, 30 \mathrm{~s}\right)$, and each sample was analyzed in triplicate. The primers used were as follows: IL- $1 \alpha, 5$ '-TGAAGCCGAGCCTCAAGATGAAG-3' and 5'-GGATGCCTGGTCACACTCAGAG-3'; IL1 $\beta, 5^{\prime}$-ACAGTGGCAATGAGGATGACTTGT-3' and 5'-TGTAGTGGTGGTCGGAGATTCGTA-3'; IL1Ra, 5' -TGTGCCTGTCCTGTGTCAAGTC-3 ' and 5 '-GCCACTGTCTGAGCGGATGAAG-3'; IL18, 5'-GCCTGGACAGTCAGCAAGGAATT-3' and 5'-GCGAGAGGAAGCGATCTGGAAG-3'; IL36Ra，5'-GGAAGCCAGTGCCTGTCATGTG-3' and 5 '-TCCCGCCGGTAGAAGGTGAAG-3 '; IL-36 $\alpha, 5$ '-CGAGGAAGGACCGTATGT-3' and 5 ' -TGGCTGTGGTAGAAGAGAA-3 '; IL-37, 5'-GCTCTGAGGACTGGGAAAAAGAT-3' and 5 '-TCACCTTTGGACTTGTGTGAAC-3 '; IL36ß, 5' -TTCCTAGCCTCCTCACCACCATC-3' and 5'-CCACTCAGGACCCACACCATCT-3'; IL$36 \gamma, 5$ '-TGGTTCATTGCCTCCTCCAAGAGA-3' and 5'-GCCACATCCTGTTCTTCAGTTGCT-3'; IL-38, 5'-GCCACACGCTTCACCTTCTTCC-3' and 5'-ACGCAGTTTCCTGTCTCCCTACC-3'; IL-33, 5'-GAAGCTCCGCTCTGGCCTTATG-3' and 5'-CTGTTGACAGGCAGCGAGTACC-3'; GAPDH, 5'-TCGGAGTCAACGGATTTGGT-3' and 5'-TTCCCGTTCTCAGCCTTGAC-3'. Data were normalized to GAPDH mRNA level, and the formula $2^{-\Delta \Delta C t}$ was used to analyze fold change of mRNA expression.

\section{Statistical analysis}

The analysis was performed with SPSS 19.0 and GraphPad Prism 5.0 for Windows. For statistical analysis, the levels of cytokines below the detection limits (Table S1) were arbitrarily assigned the values corresponding to the minimum limits. Data were expressed as mean \pm SD and median (interquartile range). Differences in percentages were evaluated using chi-square analysis or Fisher's exact tests. Differences among the groups in measured values were analyzed using the one-way ANOVA with the least significant difference test for post-hoc multiple comparisons, Kruskal-Wallis test, and Mann-Whitney $U$ test as appropriate. The bleeding scale of patients was calculated by the sum of age scale and bleeding manifestation scale (26). P values less than 0.05 were considered statistically significant.

\section{Results}

\section{Patient characteristics}

Patients newly diagnosed with primary ITP ( $\mathrm{n}=17)$ showed 
Table 1 Demographics, bleeding scores, disease duration and routine haematological parameters in primary ITP, SLE-TP, SLE-NTP patients, and healthy controls

\begin{tabular}{|c|c|c|c|c|}
\hline Variables & ITP $(n=17)$ & SLE-TP $(n=17)$ & SLE-NTP $(n=19)$ & Controls $(n=10)$ \\
\hline \multicolumn{5}{|l|}{ Sex } \\
\hline Female & 10 & 15 & 15 & 6 \\
\hline Male & 7 & 2 & 4 & 4 \\
\hline Duration of disease (thrombocytopenia >12 months) & $7 / 17$ & $4 / 17$ & - & - \\
\hline Platelet count $\left(\times 10^{9} / \mathrm{L}\right)$, median [range] & 9 [1-22] & 66 [29-95] & $183[104-327]$ & 247 [185-309] \\
\hline Absolute lymphocyte count $\left(\times 10^{\circ} / L\right)$, mean $\pm S D$ & $1.83 \pm 0.58^{\#}$ & $0.91 \pm 0.67^{*}$ & $1.03 \pm 0.53^{*}$ & $2.14 \pm 0.39$ \\
\hline Absolute neutrophil count $\left(\times 10^{9} / \mathrm{L}\right)$, mean $\pm \mathrm{SD}$ & $5.83 \pm 3.17^{\# \star}$ & $3.29 \pm 2.62$ & $4.32 \pm 2.47$ & $3.3 \pm 1.24$ \\
\hline
\end{tabular}

*, P<0.05 compared to health control; \#, P<0.05 compared to SLE-TP. ITP, immune thrombocytopenia; SLE-TP, systemic lupus erythematosus-associated thrombocytopenia; SLE-NTP, SLE without thrombocytopenia.

a median platelet count of $9 \times 10^{9} / \mathrm{L}$ and range of $1-22 \times 10^{9} / \mathrm{L}$; SLE-TP $(n=17)$ showed a median platelet count of $66 \times 10^{9} / \mathrm{L}$ and range of $29-95 \times 10^{9} / \mathrm{L}$; SLE-NTP patients $(\mathrm{n}=19)$ had a median platelet count of $183 \times 10^{9} / \mathrm{L}$ with a range of $104-327 \times 10^{9} / \mathrm{L}$. The age and sex-matched healthy controls $(\mathrm{n}=10)$ had a median platelet count of $247 \times 10^{9} / \mathrm{L}$ with a range of $185-309 \times 10^{9} / \mathrm{L}$. Characteristics of the cases enrolled in the study are shown in Table 1. No significant differences were found in age and sex distributions among the four groups $(\mathrm{P}>0.05)$. The bleeding score at initial diagnosis was higher in ITP patients than in SLE-TP patients, and the absolute lymphocyte count was decreased significantly in SLE-TP and SLE-NTP patients compared to ITP and healthy controls. The absolute neutrophil count was increased significantly in ITP patients compared with SLE-TP patients and healthy controls. However, there was no significant difference among SLE-TP patients, SLENTP patients, and healthy controls.

\section{Serum IL-1 family cytokines in primary ITP patients, SLE-TP patients, and SLE-NTP patients correlate with disease activity}

To understand the serum IL-1 cytokines profile in ITP, SLE-TP, and SLE-NTP patients, 11 cytokines including IL-1 $\alpha$, IL-1 $\beta$, IL-1Ra, IL-18, IL-36Ra, IL-36 $\alpha$, IL-37, IL-

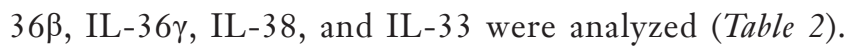
While the majority of IL-1 $\alpha$ levels were below the detection limits of the assay (data not shown), as shown in Figure 1, the secretion of serum IL- $1 \beta$, IL-18, IL-36 $\alpha$, IL-36 $\beta$, IL$36 \gamma$, and IL-33 were significantly decreased in primary ITP patients compared to SLE-TP, SLE-NTP patients. However, there were no significant differences between the SLE-NTP patients and SLE-TP patients/healthy controls (P>0.05), and except for IL-33, there were no significant differences between the SLE-TP patients and healthy controls.

As shown in Figure 2, IL-1Ra and IL-36Ra were increased in primary ITP and SLE-TP patients compared to healthy controls without significant differences. However, there was a significant difference in the secretion of IL-1Ra between the primary ITP and SLE-TP patients, and compared with the controls, IL-37 and IL-38 were decreased significantly in primary ITP patients and SLE-TP patients. The level of IL-37 was also increased significantly in SLE-NTP patients, compared with SLE-TP patients.

\section{The secretion of serum IL-37 and IL-36 correlated positively to platelet count in primary ITP and SLE-NTP patients, respectively}

A positive correlation was found between IL-37 and platelet count in primary ITP $(\mathrm{r}=0.617, \mathrm{P}=0.008$, Figure $3 A)$. No significant correlation was found between IL-1 cytokines and platelet count in SLE-TP patients, but a significant positive correlation was found between IL-36 $\alpha$ and platelet count in SLE-NTP patients $(\mathrm{r}=0.673, \mathrm{P}=0.002$, Figure 3B). 
Table 2 Serum IL-1 family cytokines levels of primary ITP, SLE-TP, and SLE-NTP patients (pg/mL)

\begin{tabular}{|c|c|c|c|c|}
\hline Variables & ITP $(n=17)$ & SLE-TP $(n=17)$ & SLE-NTP $(n=19)$ & Health controls $(n=10)$ \\
\hline IL-1F3 (IL-1Ra) & $57.61(38.23-104.74)^{\#}$ & $21.3(10.03-75.62)$ & $24.12(12.58-48.48)$ & $31.11(19.84-55.1)$ \\
\hline IL-1F4 (IL-18) & $184.66(113.92-334.54)^{\star \#}$ & 6,935.64 (281.53-9,297.78) & $8,847.64(5,663.6-10,464.8)$ & $7,497.2(5,967.43-8,144.15)$ \\
\hline IL-1F5 (IL-36Ra) & $1,103.8(406.95-4,497.75)$ & $1,150.49(131.35-3,301.73)$ & $4,073.22(1,884.32-4,863.08)^{\star}$ & 246.05 (77.23-997.78) \\
\hline IL-1F7 (IL-37) & $23.49(20-28.35)^{\star \#}$ & $30.29(22.06-38.67)^{\star}$ & $44.67(30.36-91.39)^{\#}$ & $83.96(27.1-238.27)$ \\
\hline IL-1F8 (IL-36ß) & $1.04(0.5-1.28)^{\star \#}$ & $10.97(2.56-35.15)$ & $17.26(4.62-30.25)$ & $19.79(16.58-38.93)$ \\
\hline 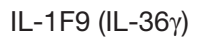 & $20(20-33.25)^{\star \#}$ & 1,151.51 (34.32-1,422.48) & 1,309.63 (742.73-2,276.75) & $2,074.9(1,136.68-2,649.83)$ \\
\hline IL-1F10 (IL-38) & $36.84(29.89-47.16)^{*}$ & $36.9(29.1-68.13)^{\star}$ & $47.21(35.73-92.29)^{*}$ & $569.97(366.32-691.12)$ \\
\hline
\end{tabular}

Data are presented as median (interquartile range). *, P $<0.05$ compared to health control; \#, $\mathrm{P}<0.05$ compared to SLE-TP. ITP, immune thrombocytopenia; SLE-TP, systemic lupus erythematosus-associated thrombocytopenia; SLE-NTP, SLE without thrombocytopenia.
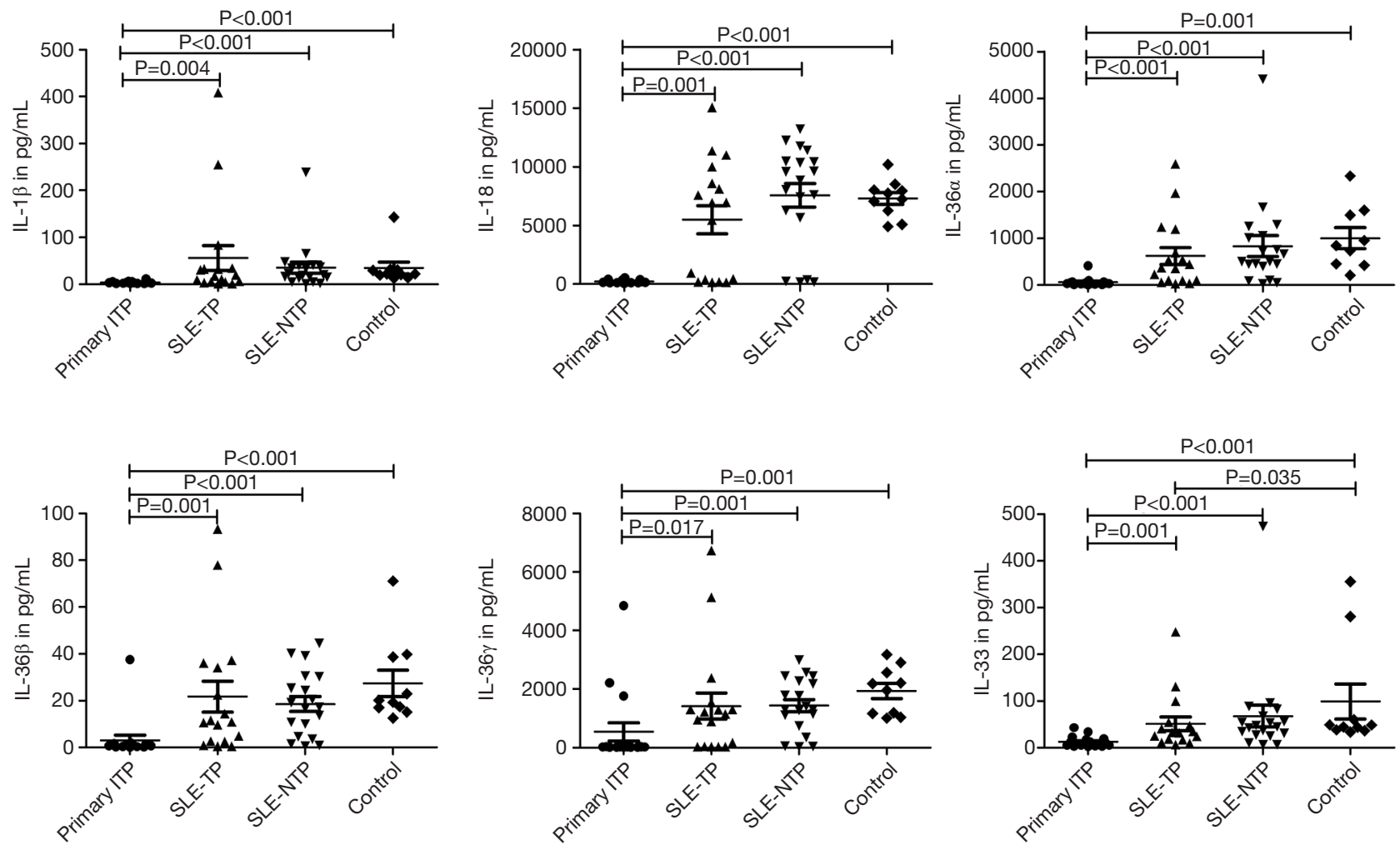

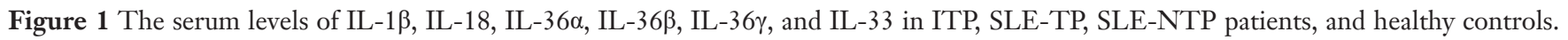

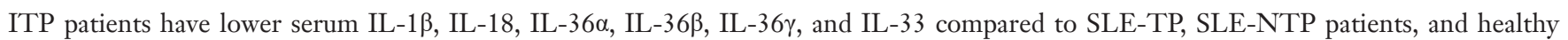

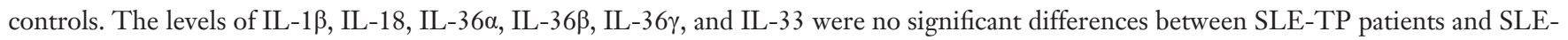
NTP patients. The level of IL-33 was increased significantly in healthy controls than SLE-TP patients. The P values less than 0.05 were labeled in the figure. ITP, immune thrombocytopenia; SLE-TP, systemic lupus erythematosus-associated thrombocytopenia; SLE-NTP, SLE without thrombocytopenia. 

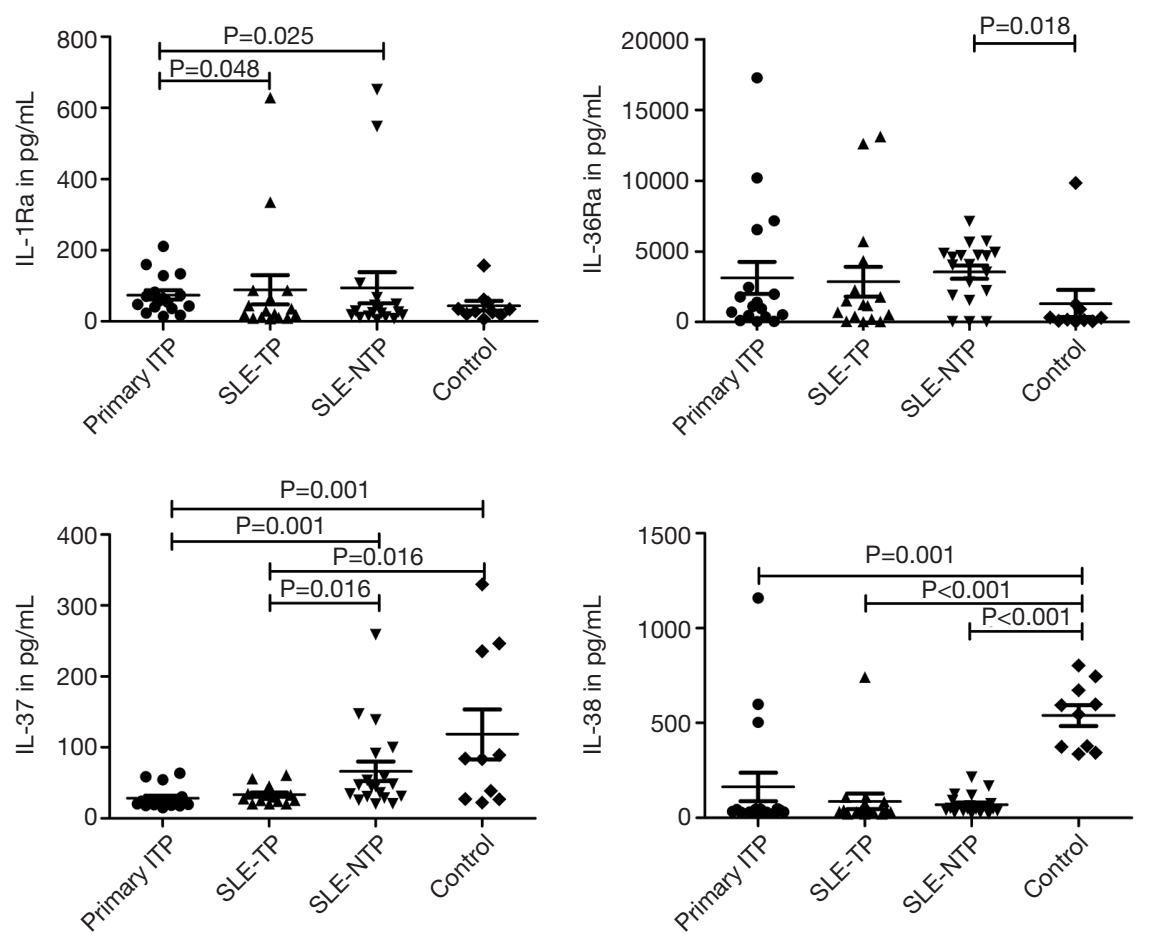

Figure 2 The levels of IL-1Ra, IL-36Ra, IL-37, and IL-38 in ITP, SLE-TP, SLE-NTP patients, and healthy controls. ITP patients have lower IL-1Ra than SLE-TP and SLE-NTP patients. The level of IL-37 was lower in ITP and SLE-TP patients compared to SLE-NTP patients and healthy controls. The level of IL-38 was lower in ITP, SLE-TP, and SLE-NTP patients compared to healthy controls. The $\mathrm{P}$ values less than 0.05 were labeled in the figure. ITP, immune thrombocytopenia; SLE-TP, systemic lupus erythematosus-associated thrombocytopenia; SLE-NTP, SLE without thrombocytopenia.
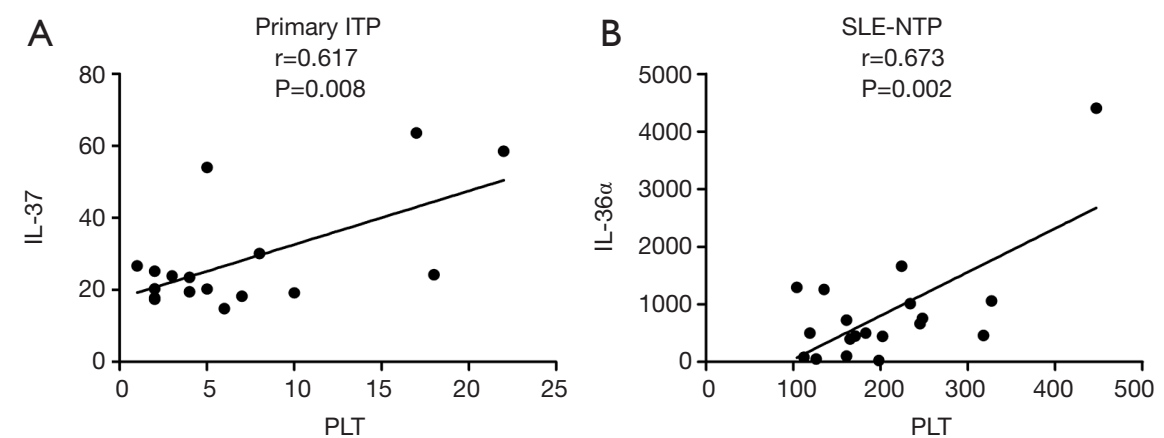

Figure 3 Serum IL-37 and IL-36a correlated with platelet count. (A) Serum IL-37 positively correlated with platelet count in ITP patients. (B) Serum IL-36 $\alpha$ positively correlated with platelet count in SLE-NTP patients. The P values less than 0.05 were labeled in the figure. ITP, immune thrombocytopenia; SLE-NTP, systemic lupus erythematosus without thrombocytopenia; PLT, platelet count. 

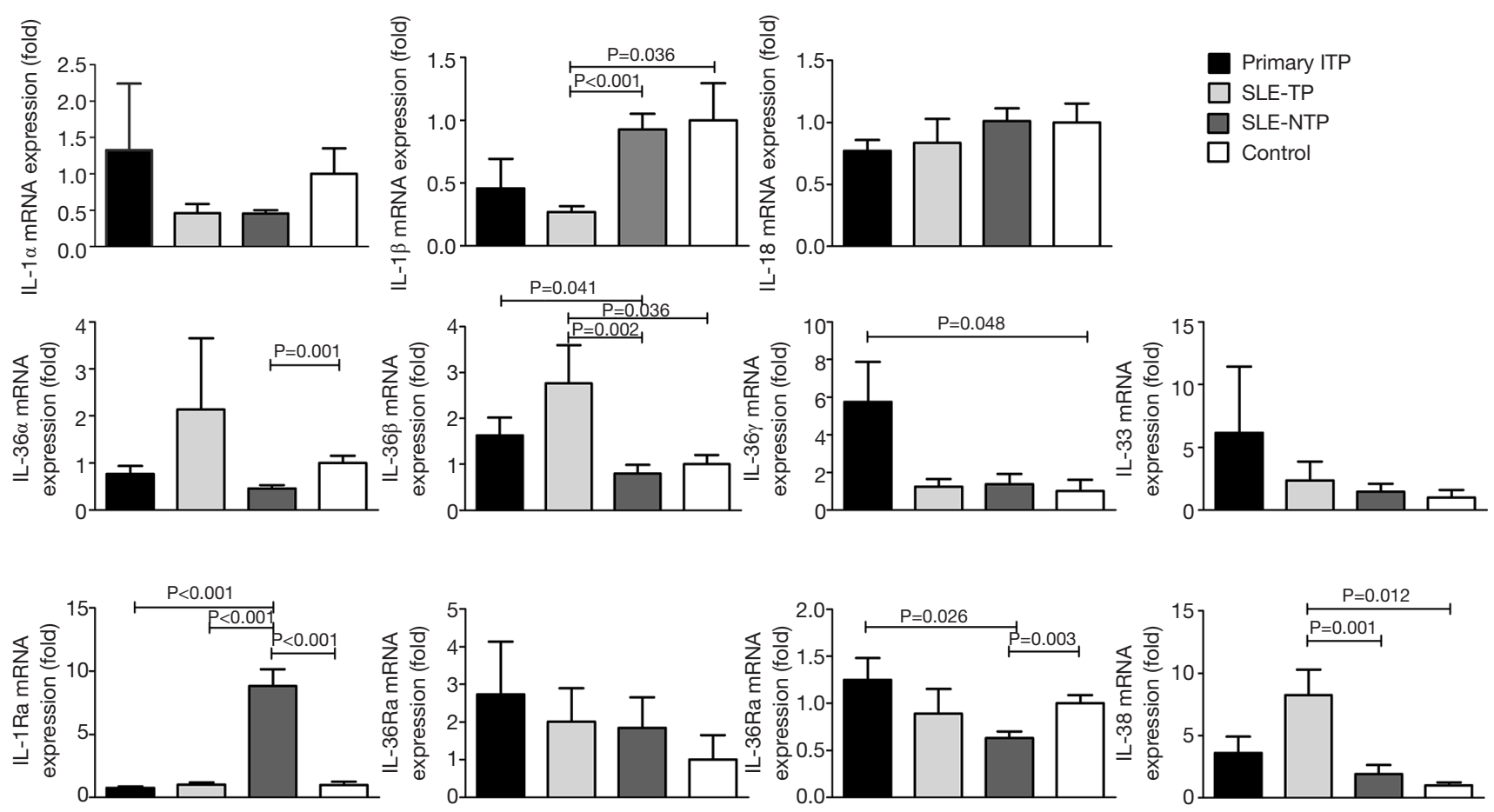

Figure 4 The mRNA expression of IL-1 cytokines in ITP, SLE-TP, SLE-NTP patients, and healthy controls. There were no significant differences between ITP and SLE-TP patients. The expression of IL-1 $\beta$ mRNA was decreased significantly in SLE-TP patients compared to SLE-NTP patients and healthy controls. The expression of IL-36 $\beta$ and IL-38 mRNA were increased significantly in SLE-TP patients

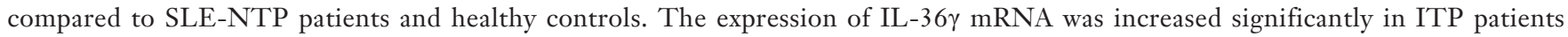
than healthy controls. The expression of IL-1Ra mRNA was increased significantly in SLE-NTP patients than ITP, SLE-TP patients, and healthy controls. The $\mathrm{P}$ values less than 0.05 were labeled in the figure. ITP, immune thrombocytopenia; SLE-TP, systemic lupus erythematosus-associated thrombocytopenia; SLE-NTP, SLE without thrombocytopenia.

\section{The change of IL-1 mRNA expression between primary ITP patients and SLE-TP patients are not like those observed at the protein level}

To better understand the expression of IL-1 cytokines in primary ITP, SLE-TP, and SLE-NTP patients, we next analyzed the mRNA expression of IL-1 cytokines. The expression of IL-1 $\beta$ mRNA was decreased significantly in SLE-TP patients as compared with those with SLE-NTP and healthy controls. However, IL-36 $\beta$ and IL-38 mRNA expression were increased significantly in SLE-TP patients compared with SLE-NTP and controls. Also, there were no significant differences between primary ITP patients and SLE-TP patients (Figure 4). The expression patterns of IL-1 mRNA expression were not like those observed at the protein levels in ITP patients and SLE-TP patients.

\section{Discussion}

ITP is an autoimmune disease characterized by abnormal cell immunity (27), and abnormal cytokines play an important role in its pathogenesis. The role of IL-1 family cytokines in the development of autoinflammatory and autoimmune diseases has gained extra attention because of their potential therapeutic targets. While IL-1 gene polymorphisms have been reported to be associated with ITP and SLE (28-31), few studies investigate IL-1 cytokines expression in thrombocytopenia, and the majority of these significant cytokines have not been identified in ITP and SLE. Increasing evidence suggests that an imbalance of immune regulation mechanisms, such as Th1/Th2 imbalance, Treg/Th17 imbalance, proinflammatory cytokines, and anti-inflammatory cytokines 
imbalance, contributes to the maintenance of immune homeostasis $(32,33)$. Increased numbers of Th17 cells have been reported in both ITP and SLE. Moreover, IL-1 may participate in the development of Th17 cells $(24,25)$. Intriguingly, we observed a down-regulation of IL-1 $\beta$, IL-

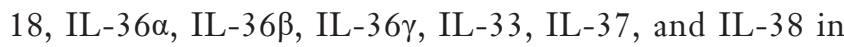
ITP patients compared to healthy controls. However, Shan et al. (23) have reported that the serum level of IL-18 was significantly increased in ITP patients. The level of IL33 in serum in our study was significantly down-regulated in ITP patients, which is following previous reports (34). IL-18 is best known for its role in Th1 immune responses, whereas IL-33 has been strongly associated with Th2 responses. All these findings support the hypothesis that IL-1 cytokines participate in the pathogenesis of ITP. Li et al. (34) also found that IL-33 mRNA expression did not differ in ITP patients than in healthy controls. In our study, not only IL-33 mRNA, but also IL- $1 \alpha$, IL- $1 \beta$, IL-1Ra, IL18, IL-36Ra, IL-36 $\alpha$, IL-36 $\beta$, IL-37, and IL-38 mRNA expression showed no significant differences in ITP patients compared to healthy controls. Our findings also indicate that no genetic variants exist in the IL- $1 \alpha$, IL- $1 \beta$, IL-1Ra, IL-18, IL-36Ra, IL-36 $\alpha$, IL-36ק, IL-37, IL-33, and IL-38 gene in ITP patients.

The derangement of immune regulation also contributes to SLE, and many studies have reported the role of IL-1 cytokines in SLE (35-38). Italiani (35) and Mende (36) found that serum IL-18 was higher in SLE than the healthy controls, although this was not seen in our study. Decreased serum IL-36Ra and increased serum IL-36 $\alpha$,

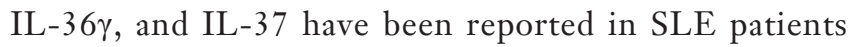
$(37,38)$, which, too, does not follow our results. Different detection methods and patient grouping may have led to these disparate test results. We first detected IL-1 cytokines in ITP, SLE-TP, and SLE-NTP patients. While antibodies cause both ITP and SLE-TP against platelets, the mechanism which differentiates these conditions is unknown. Differences in the peripheral blood absolute lymphocyte count and neutrophil count of ITP and SLETP patients in our study suggests a different cellular immunity. The assessment of IL-1 cytokines in ITP patients in comparison with those with SLE-TP and SLE-NTP

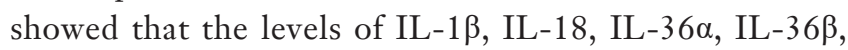

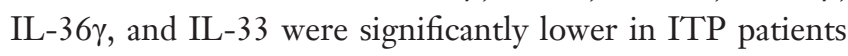
than SLE-TP and SLE-NTP patients. However, there were no significant differences in the levels of IL-1 $\beta$, IL-

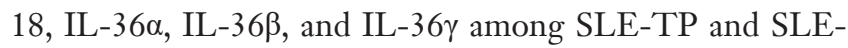
NTP patients and healthy controls. All data revealed several differences in the immunopathogenesis between ITP and SLE-TP/SLE-NTP patients. These findings indicate

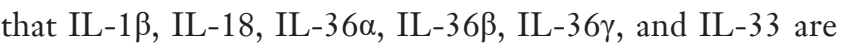
involved in the pathogenesis of ITP but not SLE, including SLE-TP and SLE-NTP. Although there was no significant difference in the level of serum IL-37 between ITP and SLE-TP patients, a significant positive correlation was found between IL-37 and platelet count in ITP patients.

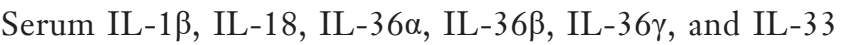
could be considered potential biomarkers that discriminate between ITP and SLE-TP patients.

Interestingly, there were no significant differences in the expression of IL-1 mRNA between ITP patients and SLE-TP patients. The change of mRNA expression of IL-1 cytokines in ITP and SLE-TP patients was not similar to those observed at the protein levels. These results lead us to conclude that post-transcriptional regulation, such as microRNAs, may take part in the pathogenesis of ITP and SLE-TP patients.

In conclusion, the present study indicates that parts of the IL- 1 cytokines, such as serum IL-1 $\beta$, IL-18, IL-36 $\alpha$, IL-36 $\beta$,

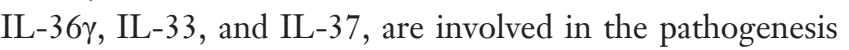
of ITP. The protein levels of IL-1 $\beta$, IL-18, IL-36 $\alpha$, IL-36 $\beta$, IL-36\%, and IL-33 rather than mRNA levels could be considered as the biomarkers to differentiate SLE-TP from ITP patients. Further investigation of the role of IL-1 cytokines in autoimmune and autoinflammation function is warranted.

\section{Acknowledgments}

This study was conducted at the Institute of Clinical Science of Zhongshan Hospital, Fudan University. We sincerely thank all the staff and participants for their important contributions.

Funding: This work was supported by grants from the National Natural Science Foundation of China (81870098, 81600090,81500090 , and 81470282), Program of the Shanghai Academic/Technology Researcher Leader (20XD1401000), Shanghai Health Bureau Funding (201840351 and 20144Y0194), and the Science and Technology Commission of Shanghai Municipality (18ZR1407200). Permission was obtained from all authorities to list them in this section.

\section{Footnote}

Reporting Checklist: The authors have completed the MDAR 
reporting checklist. Available at http://dx.doi.org/10.21037/ atm-20-4729

Data Sharing Statement: Available at http://dx.doi. org/10.21037/atm-20-4729

Conflicts of Interest: All authors have completed the ICMJE uniform disclosure form (available at http://dx.doi. org/10.21037/atm-20-4729). The authors have no conflicts of interest to declare.

Ethical Statement: The authors are accountable for all aspects of the work in ensuring that questions related to the accuracy or integrity of any part of the work are appropriately investigated and resolved. The study was in accordance with the ethical standards formulated in the Helsinki Declaration (as revised in 2013) and was approved by the respective local Medical Ethics Committees of Zhongshan Hospital of Fudan University (approval No. B2013-003R). Written informed consent was obtained from each patient before their being included in the study.

Open Access Statement: This is an Open Access article distributed in accordance with the Creative Commons Attribution-NonCommercial-NoDerivs 4.0 International License (CC BY-NC-ND 4.0), which permits the noncommercial replication and distribution of the article with the strict proviso that no changes or edits are made and the original work is properly cited (including links to both the formal publication through the relevant DOI and the license). See: https://creativecommons.org/licenses/by-nc-nd/4.0/.

\section{References}

1. Provan D, Arnold DM, Bussel JB, et al. Updated international consensus report on the investigation and management of primary immune thrombocytopenia. Blood Adv 2019;3:3780-817.

2. Cooper N, Ghanima W. Immune Thrombocytopenia. N Engl J Med 2019;381:945-55.

3. Wu B, Wang W, Zhan Y, et al. CXCL13, CCL4, and sTNFR as circulating inflammatory cytokine markers in primary and SLE-related autoimmune hemolytic anemia. J Transl Med 2015;13:112.

4. Velo-García A, Castro SG, Isenberg DA. The diagnosis and management of the haematologic manifestations of lupus. J Autoimmun 2016;74:139-60.

5. Cines DB, Bussel JB, Liebman HA, et al. The ITP syndrome: pathogenic and clinical diversity. Blood 2009; 113:6511-21.

6. Aringer M, Costenbader K, Daikh D, et al. 2019 European League Against Rheumatism/American College of Rheumatology Classification Criteria for Systemic Lupus Erythematosus. Arthritis Rheumatol 2019;71:1400-12.

7. Altintas A, Ozel A, Okur N, et al. Prevalence and clinical significance of elevated antinuclear antibody test in children and adult patients with idiopathic thrombocytopenic purpura. J Thromb Thrombolysis 2007;24:163-8.

8. Kuwana M, Kaburaki J, Okazaki Y, et al. Two types of autoantibody-mediated thrombocytopenia in patients with systemic lupus erythematosus. Rheumatology (Oxford) 2006;45:851-4.

9. Lazarus AH, Ellis J, Semple JW, et al. Comparison of platelet immunity in patients with SLE and with ITP. Transfus Sci 2000;22:19-27.

10. Dinarello CA. Interleukin-1 in the pathogenesis and treatment of inflammatory diseases. Blood 2011;117:3720-32.

11. Fields JK, Gunther S, Sundberg EJ. Structural basis of IL-1 family cytokine signaling. Front Immunol 2019;10:1412.

12. Baker KJ, Houston A, Brint E. IL-1 family members in cancer; two sides to every story. Front Immunol 2019;10:1197.

13. Dinarello CA, Simon A, van der Meer JW. Treating inflammation by blocking interleukin-1 in a broad spectrum of diseases. Nat Rev Drug Discov 2012;11:633-52.

14. Gabay C, Lamacchia C, Palmer G. IL-1 pathways in inflammation and human diseases. Nat Rev Rheumatol 2010;6:232-41.

15. Dinarello CA. Immunological and inflammatory functions of the interleukin-1 family. Annu Rev Immunol 2009;27:519-50.

16. Simon A, van der Meer JW. Pathogenesis of familial periodic fever syndromes or hereditary autoinflammatory syndromes. Am J Physiol Regul Integr Comp Physiol 2007;292:R86-98.

17. Masters SL, Simon A, Aksentijevich I, et al. Horror autoinflammaticus: the molecular pathophysiology of autoinflammatory disease $\left(^{*}\right)$. Annu Rev Immunol 2009;27:621-68.

18. Ridker PM, MacFadyen JG, Thuren T, et al. Effect of interleukin-1beta inhibition with canakinumab on incident lung cancer in patients with atherosclerosis: exploratory results from a randomised, double-blind, placebo- 
controlled trial. Lancet 2017;390:1833-42.

19. Wu TC, Xu K, Martinek J, et al. IL1 Receptor antagonist controls transcriptional signature of inflammation in patients with metastatic breast cancer. Cancer Res 2018;78:5243-58.

20. Calvani N, Tucci M, Richards HB, et al. Th1 cytokines in the pathogenesis of lupus nephritis: the role of IL-18. Autoimmun Rev 2005;4:542-8.

21. Liang $\mathrm{D}, \mathrm{Ma} \mathrm{W}$, Yao C, et al. Imbalance of interleukin 18 and interleukin 18 binding protein in patients with lupus nephritis. Cell Mol Immunol 2006;3:303-6.

22. Tucci M, Quatraro C, Lombardi L, et al. Glomerular accumulation of plasmacytoid dendritic cells in active lupus nephritis: role of interleukin-18. Arthritis Rheum 2008;58:251-62.

23. Shan NN, Zhu XJ, Peng J, et al. Interleukin 18 and interleukin 18 binding protein in patients with idiopathic thrombocytopenic purpura. Br J Haematol 2009; 144:755-61.

24. Sutton C, Brereton C, Keogh B, et al. A crucial role for interleukin (IL)-1 in the induction of IL-17-producing T cells that mediate autoimmune encephalomyelitis. J Exp Med 2006;203:1685-91.

25. Acosta-Rodriguez EV, Napolitani G, Lanzavecchia A, et al. Interleukins 1 beta and 6 but not transforming growth factor-beta are essential for the differentiation of interleukin 17-producing human T helper cells. Nat Immunol 2007;8:942-9.

26. Liu XG, Bai XC, Chen FP, et al. Chinese guidelines for treatment of adult primary immune thrombocytopenia. Int J Hematol 2018;107:615-23.

27. Ji X, Zhang L, Peng J, et al. T cell immune abnormalities in immune thrombocytopenia. J Hematol Oncol 2014;7:72.

28. Pesmatzoglou M, Lourou M, Goulielmos GN, et al. DNA methyltransferase $3 \mathrm{~B}$ gene promoter and interleukin-1 receptor antagonist polymorphisms in childhood immune thrombocytopenia. Clin Dev Immunol 2012;2012:352059.

Cite this article as: Zhan Y, Cheng L, Wu B, Ji L, Chen P, Li F, Cao J, Ke Y, Yuan L, Min Z, Sun L, Chen H, Hua F, Cheng Y. Interleukin (IL)-1 family cytokines could differentiate primary immune thrombocytopenia from systemic lupus erythematosusassociated thrombocytopenia. Ann Transl Med 2021;9(3):222. doi: $10.21037 /$ atm-20-4729
29. Yadav DK, Tripathi AK, Gupta D, et al. Interleukin-1B (IL-1B-31 and IL-1B-511) and interleukin-1 receptor antagonist (IL-1Ra) gene polymorphisms in primary immune thrombocytopenia. Blood Res 2017;52:264-9.

30. $\mathrm{Xu} \mathrm{W,} \mathrm{Liu} \mathrm{Y,} \mathrm{Ye} \mathrm{D.} \mathrm{Association} \mathrm{between} \mathrm{IL-33} \mathrm{gene}$ polymorphisms (rs1929992, rs7044343) and systemic lupus erythematosus in a Chinese Han population. Immunol Invest 2016;45:575-83.

31. Mohammadoo-Khorasani M, Salimi S, Tabatabai E, et al. Interleukin-1 $\beta$ (IL-1 $\beta$ ) \& IL-4 gene polymorphisms in patients with systemic lupus erythematosus (SLE) \& their association with susceptibility to SLE. Indian J Med Res 2016;143:591-6.

32. Shao X, Wu B, Cheng L, et al. Distinct alterations of CD68(+)CD163(+) M2-like macrophages and myeloidderived suppressor cells in newly diagnosed primary immune thrombocytopenia with or without CR after highdose dexamethasone treatment. J Transl Med 2018;16:48.

33. Cheng L, Liu C, Li F, et al. The prediction value of Treg cell subtype alterations for glucocorticoid treatment in newly diagnosed primary immune thrombocytopenia patients. Thromb Res 2019;181:10-16.

34. Li PP, Zhang XM, Yuan D, et al. Decreased expression of IL-33 in immune thrombocytopenia. Int Immunopharmacol 2015;28:420-4.

35. Italiani P, Manca ML, Angelotti F, et al. IL-1 family cytokines and soluble receptors in systemic lupus erythematosus. Arthritis Res Ther 2018;20:27.

36. Mende R, Vincent FB, Kandane-Rathnayake R, et al. Analysis of serum interleukin (IL)-1 $\beta$ and IL-18 in systemic lupus erythematosus. Front Immunol 2018;9:1250.

37. Mai SZ, Li CJ, Xie XY, et al. Increased serum IL$36 \alpha$ and IL-36 $\gamma$ levels in patients with systemic lupus erythematosus: association with disease activity and arthritis. Int Immunopharmacol 2018;58:103-8.

38. Wu GC, Li HM, Wang JB, et al. Elevated plasma interleukin-37 levels in systemic lupus erythematosus patients. Lupus 2016;25:1377-80. 
Supplementary

Table S1 Detection range of IL-1 family cytokines

\begin{tabular}{|c|c|c|}
\hline Variables & Lower limits of detection, $\mathrm{pg} / \mathrm{mL}$ & Upper limits of detection, $\mathrm{pg} / \mathrm{mL}$ \\
\hline IL-1F1 (IL-1 $\alpha)$ & 0.5 & 1,000 \\
\hline IL-1F2 (IL-1 $1 \beta)$ & 1.0 & 2,000 \\
\hline IL-1F3 (IL-1Ra) & 2.5 & 4,000 \\
\hline IL-1F4 (IL-18) & 7.0 & 10,000 \\
\hline IL-1F5 (IL-36Ra) & 20.0 & 100,000 \\
\hline IL-1F6 (IL-36 $\alpha)$ & 20.0 & 100,000 \\
\hline IL-1F7 (IL-37) & 20.0 & 100,000 \\
\hline IL-1F8 (IL-36ß) & 0.5 & 1,000 \\
\hline 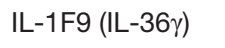 & 20.0 & 100,000 \\
\hline IL-1F10 (IL-38) & 20.0 & 100,000 \\
\hline IL-1F11 (IL-33) & 7.0 & 10,000 \\
\hline
\end{tabular}

